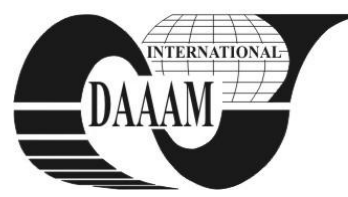

Annals of DAAAM for 2011 \& Proceedings of the 22nd International DAAAM Symposium, Volume 22, No. 1, ISSN 1726-9679 ISBN 978-3-901509-83-4, Editor B. Katalinic, Published by DAAAM International, Vienna, Austria, EU, 2011 Make Harmony between Technology and Nature, and Your Mind will Fly Free as a Bird

\title{
CREATION OF 3D-CAD VIRTUAL MODEL FOR RAPID PROTOTYPING MICROTECHNOLOGIES USING SELECTIVE LASER SINTERING
}

\author{
GHEORGHE, I[on] G[heorghe]; CIOBOTA, N[astase] - D[an] \& PACIOGA, A[drian]
}

\begin{abstract}
The scientific paper presents the design and construction of $3 D-C A D$ virtual model, through parametric modeling using the CAD-CAM-FEA capabilities of the SolidWorks program. The article presents the design stages of the virtual model of a propeller-shaped rotor and the preprocessing methods of the part and supports for sintering preparation. The aim is to demonstrate that laser sintering can be successfully applied to rapid manufacturing of complex shaped parts which can not be obtained using conventional machining technologies. At the end of the paper the physical model of the propeller-shaped rotor obtained at our institute using an EOS M270 machine is presented, thus proving the effectiveness of the process for complex shaped parts manufacturing.
\end{abstract}

Key words: virtual model, parametric modeling, rapid prototyping, pre-processing

\section{INTRODUCTION}

Information explosion in recent decades has led to substantial changes in technologies and materials processing. There appeared new technologies which meet the diverse requirements of all producers, creating new opportunities regarding the final form of the parts which is becoming increasingly complex. Direct metal laser sintering of metallic powders (DMLS) is already known as a technology which provides the fastest path from the idea to product market launch. This technology can be used for rapid and flexible implementation of parts with minimal production costs, directly from electronic virtual 3D model, in any phase of the manufacturing cycle (Shellabear, 2004). The method can be used for obtaining complex shaped parts on a laser sintering machine EOS M270 for metallic powder, version which includes the possibility of titanium powder processing.

Research on how to design a virtual 3D-CAD parametric model and the pre-processing stages of a small complex part for laser sintering, are presented in the frame of the paper. This research proves that the still existing distrust in using this method is not justified, because the results regarding the shape, dimensions and mechanical characteristics of obtained parts are very promising and because of the simplicity of the CAD-CAM method.

\section{CREATION OF 3D-CAD VIRTUAL MODEL}

Usually, the creation of the 3D virtual model is done by parametric 3D modeling using one of the commercial CADCAM-FEA programs.

A common feature of all computer-aided design software packages, using parametric modeling, is the work with graphic blocks. Commonly, these blocks are of two types: with implicit geometry (circular cross section holes, bevels, fillets, and roundings) and with explicit geometry (in this case the base element is the section's shape) $(* * *, 2011$ a). Construction of a geometric model starts with the basic component, which is always obtained through material addition. On this basic element the other necessary graphic blocks are than attached by adding or removing material, which will be directly or indirectly connected to the base.

The connections between the graphic blocks of a 3D virtual model are a fundamental characteristic feature of the parametric geometric modeling programs. This feature leads to automatic updates of the graphic blocks when changing the size or geometry of the basic construction element on which these are attached (connected).

The graphic building blocks with explicitly geometry are based on a $2 \mathrm{D}$ profile representing the section shape. With this $2 \mathrm{D}$ profile, one can add or remove material through a process of extrusion, revolve around an axis or displacement along a given curve. We will define the $3 \mathrm{D}$ geometric modeling stages of a propeller-shaped rotor with complex shape whose functional geometry is shown in Fig. 1.a. Parametric modeling steps are cycled as follows:

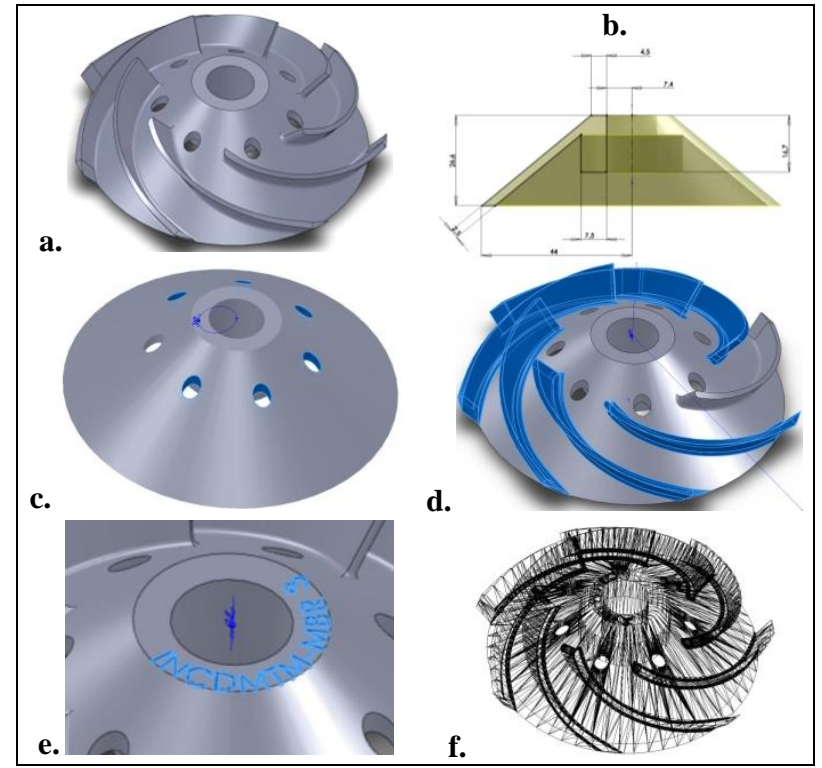

Fig. 1. The propeller-shaped rotor/3D-CAD modeling stages

\subsection{Analysis of the part in terms of functionality.}

The proposed part can't be obtained by conventional machining technologies, but can be manufactured by casting or laser sintering. The seven holes are relief holes and their position (on the surface of the part) is symmetrical in relation to the median axis of the work piece. The seven vertical protrusions with variable section are the rotor's blades and they are also symmetrical in relation to the axis of the rotor. The inner cylindrical surface is a centering surface and the adjacent flat surfaces are positioning surfaces.

\subsection{Establishing the basic graphic building element.}

The part has a circular shape, which suggests that the basic building element should be obtained by a revolution operation. 
Choosing an extrusion operation to obtain the basic building block would be a wrong choice since it implies (after extrusion) a very large number of extruded cuts. In fig. 1. b-e are presented the design stages of the propeller-shaped rotor in the SolidWorks program. Thus in figure 1.b. is shown the sketch used in the revolve operation, the result of this operation being the basic building block. On this block are then added other auxiliary graphic building blocks for achieving virtual 3D-CAD model.

\subsection{Identifying and realizing the auxiliary graphic blocks}

Auxiliary graphic blocks remaining to be done are: relief holes and the rotor blades with the subsequent fillets. The seven holes can be obtained most easily by making a hole and then multiply them in relation to the axis of the part using a circular pattern scheme (see fig. 1.c.). Achievement of seven individual holes would require allocation of additional time for quoting the diameter and position of each hole.

To obtain the rotor blades, since they have variable section, there is only one modeling version using the loft command (the union of two sections, usually of different shapes and sizes, using a curved support). After completion of the blade construction and adding the elements with default geometry (ex. fillets), the next step is their multiplication in relation to the median axis of the part (see fig. 1.d.). Finally, one can add other details such as inscriptions or other notations (see Fig. 1.e.), the finesse of the sintering process allowing their obtaining.

\subsection{Establishing the sequence of adding the graphic blocks}

After establishing the necessary building blocks in order to obtain the part, before moving on to the effective geometric modeling, the order of adding these graphic blocks must be determined. In our example the modeling order can be amended so as to achieve first the blades and then the relief holes, but there are situations when the sequence it is stricter and the order can not be changed.

\subsection{Conversion of the CAD file in STL file}

At the end of parametric design stage, in order to prepare the part for direct laser sintering process it is necessary to convert the CAD file into STL file (see fig. 1.f.). This conversion is necessary since it is the standard format adopted for the rapid prototyping industry. This format represents the three-dimensional surfaces as a set of flat triangles, and the file is containing the coordinates of the triangle vertices given by position vector data (Gheorghe, 2010).

\section{PART ORIENTATION FOR SINTERING}

For orientation and positioning of the part the program Magics 12.1 was used. This program allows modification of STL files, ensuring handling, repairing, measurement and modification of parts in STL format. To facilitate the operation of orientation, the program has preset orientations, commands for alignment with the platform, and manual controls for movement and rotation in absolute or relative coordinates. Using these tools the part can be translated, rotated, scaled, duplicated or mirrored (for non symmetrical parts) (***, 2011 b).

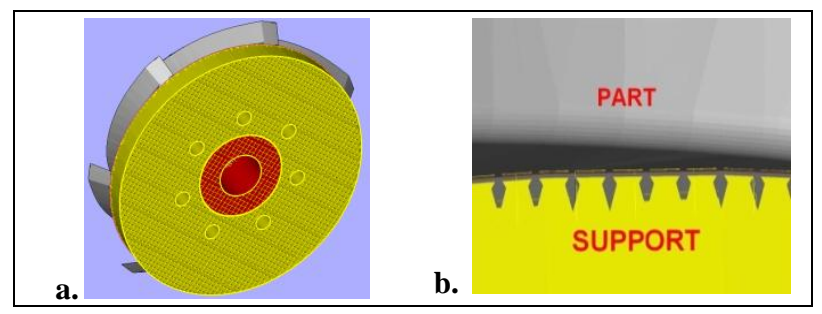

Fig. 2. Geometry of the part's supports
Also in this program are generated the supports, which confers stability to the part to be processed by sintering, and their specific geometry provides a predetermined breaking point to allow quick removal of the work piece from the platform at the end of the sintering process (see fig. 2.a. and 2.b.)

\section{PRE-PROCESS OF THE PART AND SUPPORTS}

In the next step, the part and the supports are pre-processed using the EOS RP Tools program, in which the threedimensional STL file is converted in a two-dimensional file, SLI type. This conversion is necessary because sintering occurs only through layer by layer melting (fig. 3). The virtual 3D model and the supports are divided into layers (bottom up) with a thickness of 0.02 or $0.03 \mathrm{~mm}$ depending on the material used.

After "slicing" the part, an error checking must be carried out and corrections of the file must be made if such errors are discovered. The operation is necessary as parts made in 3D parametric design software may have some minor errors, most often undetectable to the naked eye, but which can result in serious problems during the sintering operation. The part obtained by sintering at our institute is shown in fig. 4 .

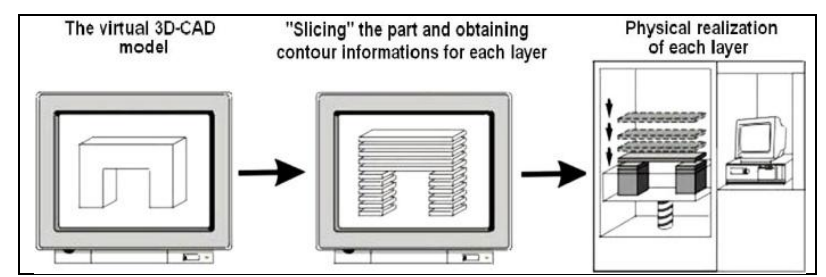

Fig. 3. Conversion of 3D STL file in 2D SLI file

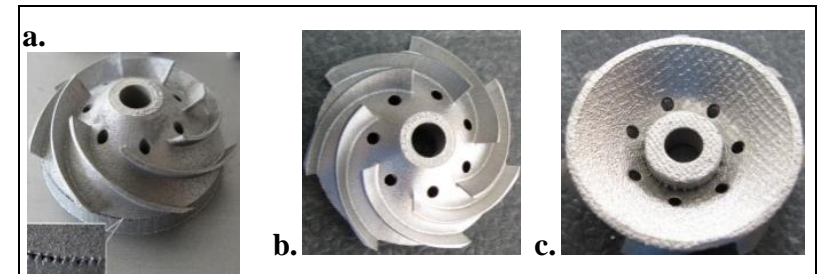

Fig. 4. The physical model of the propeller-shaped rotor

\section{CONCLUSIONS}

The carried out research aimed to establish clear design and construction stages of the virtual 3D-CAD model for a complex shaped part by using parametric modeling capabilities of SolidWorks program, and also pre-processing stages of the part and supports for sintering process. Experiments show that rapid prototyping can be successfully used for the manufacture of unique parts, accurate and of good quality. The following research will be focused on creating a method for improving the accuracy of the sintered parts.

\section{REFERENCES}

Gheorghe, Ion; et. al. (2010). Microtehnologii avansate prin prototipare rapidă cu sinterizare selectivă cu laser, Ed. CEFIN, ISBN 978-606-92267-8-0, București, pp. 160-177

Shellabear, M.; Nyrhilä, O. (2004). DMLS - Development history and state of the art, Proceedings of $4^{\text {th }}$ International conference on Laser Assisted Net Shape Engineering, Sept. 2004, Erlangen, Germany, ISBN: 3-87525-154-7, Geiger, M.; Otto, A. (Eds.), pp: 393-404, Pb. Bamberg-Meisenbach *** (2009). M270 St-Xt: Operation Manual, pp.: 6.1-6.11

*** $(2011, \mathrm{a})$. http://www.solidworks.com/ - SolidWorks: 3D CAD Design Software, Accessed on: 2011-03-12

*** (2011,b). http://www.materialise.com/ - Materialise. Rapid Prototyping and Manufacturing, Accessed on: 2011-03-01 